this day - much like the nonagenarian author. Unwavering, outspoken and somewhat self-promoting, he admittedly uses Memoirs to present his "version of the facts", but he also writes with warmth, humour and optimism. There is indeed only one Teller. John T. Finn is in the Systems Research Department, Sandia National Laboratories, Livermore, California 94551-0969, USA.

\section{A toast to the genome}

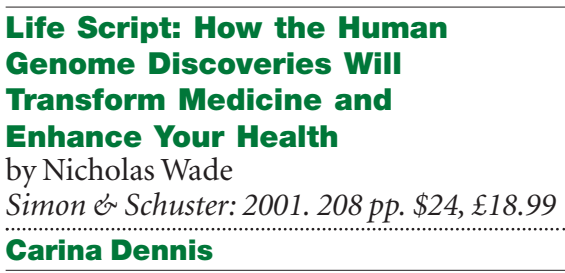

Many of the milestones along the epic journey to sequencing the human genome first came to light in the headlines of newspapers and on television. Rivals from different camps would compete for the bigger
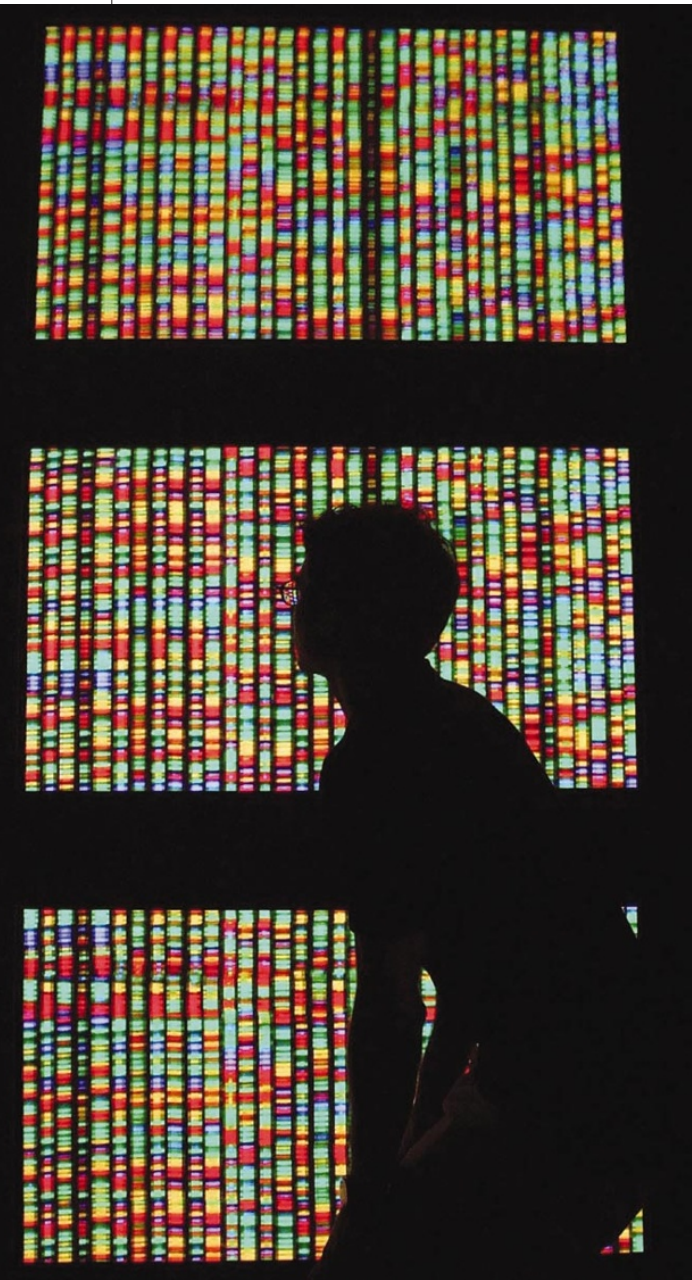

Admirable sight: an observer studies the

human genome sequence. media splash, with the elbowing intensifying in the lead up to the publication of the sequence in Nature and Science early last year. Nicholas Wade is the New York Times' journalist at the genome frontline; he draws on his own extensive reporting of the subject to bring us a book that recounts the events marking the 'dawn of the genome revolution' and illustrates how it lays the groundwork for a new era in medicine.

Wade hits his stride early in the book, portraying both the science and the drama behind the 'race', between the international consortium of academic researchers and the private venture Celera, to sequence the human genome. The story doesn't need any added spice, for it is already peppered with turf wars, public and private mêlée, vendetta and vindication. (Surely the mini-series can't be far behind.) Notably, Wade tempers the theme of the 'race', diplomatically proclaiming no clear winner, as it does indeed depend "on the yardstick applied". Nevertheless, he does not shy away from lending his own interpretation of events and their significance, and he tips plausible contenders for the Nobel prize, in the event that it is awarded for the sequencing of the human genome.

At roughly halfway, the book shifts to visions of how genomics will transform human health. At this point, the fluidity of the book is displaced by a list of the many tangents that post-genomic research will take as it strives to conquer human disease. Certainly, the examples given are timely we read of the recent discoveries of genetic variations that underpin diabetes and Crohn's disease, the design of drugs to specifically intercept the genetic perturbations of cancer, and the molecular basis for why asthma patients respond differently to a widely used drug treatment. This chapter is a tour de force of futuristic genomic medicine, but the jolting delivery and propensity for using technical concepts that are not fully explained may leave the non-biologist reader floundering.

For those tempted to put the book aside at this point, I urge you to persevere. For Wade soon resumes his lucid and evenly paced stride in chapters on two topical aspects of modern research: regenerative medicine and immortality. The author traces the quest to find the elusive means of treating stem cells so that they will eventually repair diseased tissues and rejuvenate dilapidated organs, in addition to the scientific ingenuity that could one day extend the human lifespan. Here, Wade is both entertainer and educator, sweeping the reader along with lively accounts of the experiments that are edging us closer to an era of renewable and sustainable health.

The genomics company Human Genome Sciences (HGS), and particularly its chief executive William Haseltine, feature prominently, more so than the multitude of other commercial endeavours intent on mining the genome for its treasures. In the background of the genome sequencing effort, the HGS has been busily capturing and cataloguing the expressed sequences of the genome, with the focus being on the signalling molecules that form the essential communication network between cells, and which are undoubtedly involved in cancer and other diseases. Wade's sympathetic handling of the company's efforts hint that he has great faith in their success. Nevertheless, as he notes, without publication of their findings, the jury is still out on the merits of their claims.

For those desiring a quick snapshot of how medicine is being transformed by genetic knowledge, this book is well ahead of the textbooks, but it will inevitably date as the field streaks ahead at its breakneck pace. A cautionary note is warranted regarding the genetic panacea seemingly idealized in the book. Amplifying the belief of James Watson, a pioneer of the genomic revolution, that the "biggest ethical problem we have is not using our knowledge", Wade presents a persuasive argument that the fruits of pharmacogenomics will cure all our medical malaises. This is reminiscent of arguments by its advocates that nuclear power will solve our energy crisis. I dare say that it may make our lives better, provided that we can use it without hurting ourselves in the process.

Caution aside, we have a long road yet to travel, but this book is a toast to your genome and to your good health.

Carina Dennis is Nature's Australasian correspondent.

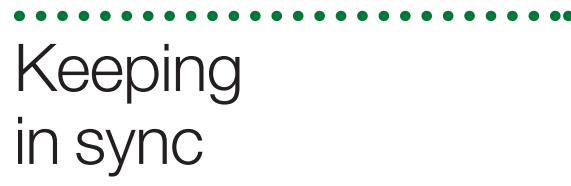

\section{Synchronization: A Universal}

Concept in Nonlinear Sciences

by Arkady Pikovsky, Michael Rosenblum \& Jürgen Kurths

Cambridge University Press: 2001. 432 pp.

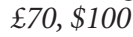

\section{William Ditto}

Have you noticed lately that most technical books make you feel as if you have wandered into the middle of a French art film dubbed in Mandarin? You don't know whether you care about the issues being addressed or whether it is worth your while to persevere. It excites me when talented scientists break free from the normally turgid prose encouraged by technical journals and write a delightful book that illuminates a subject that is so desperately in need of light. Arkady Pikovsky, Michael Rosenblum and Jürgen Kurths succeed at all levels in writing a definitive exposition of the 

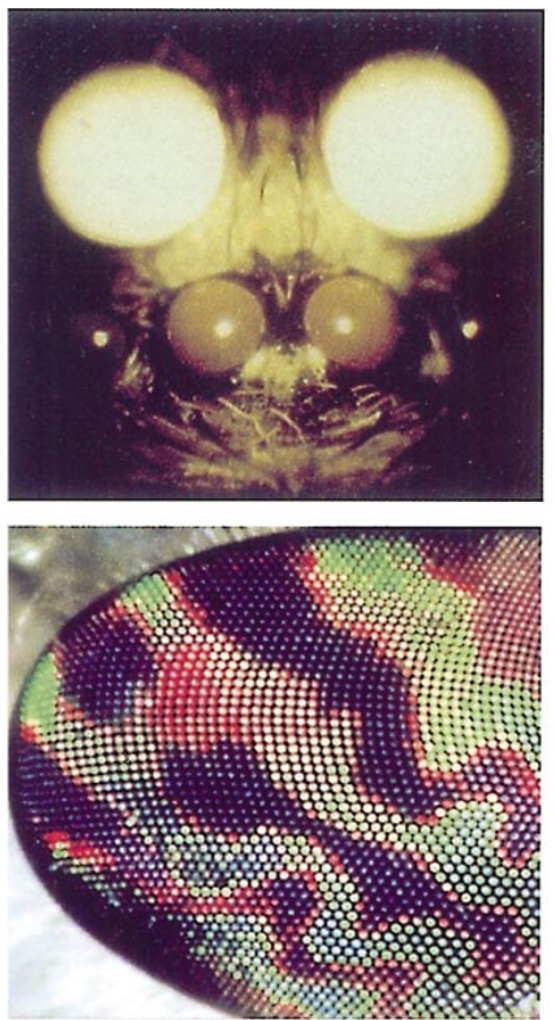

\section{Visual virtuosity}

The nocturnal wolf spider (top, left; its retina, right) has eight eyes, four of which have a mirror, or tapetum, which reflects light back through the retina. Animal Eyes by M. F. Land
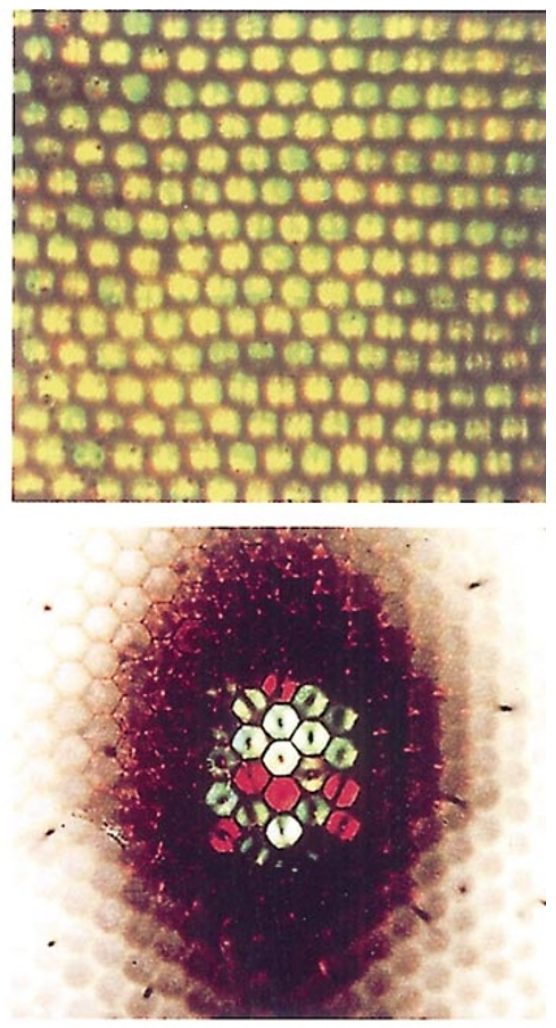

and D.-E. Nilsson (Oxford University Press, $\mathfrak{E 2 4 . 9 5 , \$ 4 5 ; ~ p b k ) ~ d i s c u s s e s ~ s u c h ~ a d a p t a t i o n s ~}$ and many more, including the visual equipment of the horsefly (below, left) and butterfly (right).

makers, menstrual cycles, circadian rhythms and chaotic synchronization. Attractive illustrations tell the story behind each example and demonstrate an important topic to full effect. There is also a section (often neglected by more reality-challenged authors) called "Detecting synchronization in experiments", which explores the consequences of when theory meets experiment. This section alone is worth the price of the book.

One-third of the way into the book, I encountered mathematical equations and, amazingly, was sufficiently motivated and had learnt enough from the previous chapters to begin to establish a meaningful relationship with the increasing complexity. I was rapidly guided through the essentials of the synchronization of driven systems, mutual synchronization, noisy synchronization, oscillatory media, phase synchronization of chaotic systems and globally coupled oscillators. These topics are liberally sprinkled with eclectic and interesting examples and with sufficient references to enable the reader to delve further into relevant questions of synchronization in a variety of fields.

The book concludes with one of the best expositions on the synchronization of chaotic systems that I have read. The sections on, for example, phase (and how it is defined) in chaotic systems, the relationships between unstable periodic orbits embedded in chaotic systems and generalizations of synchronization in complex systems (neglected by most standard treatments) are outstanding.

Clearly, the authors of Synchronization have pulled off a very difficult trick, that of writing a book that is both a definitive introduction to synchronization for the casual reader and a definitive text for researchers working in a variety of fields. Still, if I might make a suggestion for the second edition of this book - defy tradition and use pictures of smiling scientists!

William Ditto is in the Wallace H. Coulter Department of Biomedical Engineering, Georgia Tech/Emory University, 315 Ferst Drive, IBB 3306, Atlanta, Georgia 30332-0535, USA.

\section{Words of \\ climatic wisdom}

\section{The Encyclopaedic Dictionary of Environmental Change}

edited by John A. Matthews et al.

Arnold: 2001. 701 pp. $£ 125$

\section{Heike Langenberg}

Those who have attempted interdisciplinary collaboration know that one of the highest hurdles to overcome can be language. For example, by 'ventilation' an oceanographer means an influx of water masses rather than anything to do with wind or air. And words such as 'vorticity' are just jargon to non-specialists. These obstacles can make reading literature outside one's own field of research - as well as talking to colleagues from another department - anything from difficult to hazardous.

The problem is well known from the realm of foreign language. With some training in French but no Latin, at the age of about 15 my sister made an educated guess at the translation of Caesar's title De Bello Gallico and came up with "The beautiful Gaul". To avoid such potentially embarrassing pitfalls, dictionaries have long served the students of foreign languages. The Encyclopaedic Dictionary of Environmental Change aims to support students and researchers of the environmental sciences in much the same way.

But, true to its title, the book is more than just a dictionary. Short paragraphs in understandable language briefly explain the basic concept behind most terms; longer pieces of up to a page are devoted to broader fields such as the "geological record of environmental change". And references, remarkably up to date and citing a good proportion of work from this millennium, help the interested reader to dig deeper than the book can go. Not entirely true to the title, The 